shown a high incidence of chronic arachnoiditis (diagnosed by radiological criteria). For some time it has been a recognised risk of the use of the oil-soluble media (such as iophendylate). Unfortunately aspiration of the contrast medium after the examination does not necessarily give protection. The hope that water-soluble substances would prove innocuous has proved false, and many cases have been described after the use of iothalamate meglumine (Conray) and iocarmate meglumine (Dimer-X). So far, however, sodium calcium medetate (metrizamide) has proved to be safe $^{6}$ : it has been more extensively tested than any other contrast medium but no case of arachnoiditis has yet been attributed to its use.

The subdural space is very sensitive to irritants, even as benign as blood, and insufficient attention has been paid to subdural leakage as a cause of arachnoiditis. Could constrictive arachnoiditis really be a reaction in the subdural space? If this is so-and there is an association ${ }^{3}$ between "difficult" lumbar punctures and arachnoiditis-then doctors should give more consideration to postponing a radiological contrast examination in any case in which puncture of the subarachnoid space has not been clean and immediate. For the time being metrizamide seems to be the spinal contrast medium of choice, while air should be used whenever possible as an alternative, especially in children.

\footnotetext{
${ }^{1}$ Elkington, J St C, Brain, 1936, 59, 181.

${ }^{2}$ Elkington, J St C, in Modern Trends in Neurology, ed A Feiling, p 149. London, Butterworth, 1951.

3 Shaw, M D M, Russell, J A, and Grossart, K W, fournal of Neurology, Neurosurgery, and Psychiatry, 1978, 41, 97.

- Bernat, J L, Fournal of Neurology, Neurosurgery, and Psychiatry, 1976, 39, 1124.

5 Ransford, A C, and Harries, B J, fournal of Bone and foint Surgery, 1972, 54, 656 .

${ }^{6}$ Hansen, E B, Fahrenkrieg, A, and Praestholm, J, British fournal of Radio$\log y, 1978, \mathbf{5 1}, 321$
}

\section{Growing fractures of the skull}

In 1816 John Howship ${ }^{1}$ described a 9-month-old child who had developed partial absorption of the right parietal bone after a blow to the head. The names used since then to describe the condition include meningocele spuria, ${ }^{2}$ traumatic cephalhydrocele, ${ }^{3}$ traumatic ventricular cyst, ${ }^{4}$ and leptomeningeal cyst. ${ }^{5}$ More recently the term "growing fracture" has become accepted. ${ }^{6}$ There are four characteristic features: a skull fracture in infancy or early childhood; a dural tear; brain injury beneath the fracture; and subsequent enlargement of the fracture to form a cranial defect. ${ }^{7}$

Growing fracture is uncommon; one American series documented only seven patients operated on in 20 years, ${ }^{8}$ but a recent paper from London has reported 10 patients seen in 10 years. ${ }^{9}$ Most had had their initial injury before the age of 1 year and almost all by 3 ; the fracture was commonly in the parietal region. The time between injury and diagnosis was generally short; the mean interval was 15 months, the shortest two and the longest 63 months. The diagnoses in this series were made earlier than in those previously reported, ${ }^{8}$ probably because surgeons are more aware of the condition now that the criteria for its diagnosis have been established.

Patients most often present with a pulsatile swelling of the scalp, though seizures and neurological deficits such as hemiplegia may occur. Neuroradiological investigations show dilatation of the ipsilateral ventricle with midline displacement to the same side. A porencephalic cyst is generally present and often communicates with the lateral ventricle. ${ }^{8}$ In the past these features have been shown by pneumoencephalography, but CAT scanning is likely to prove valuable in making the diagnosis: it will show up not only the underlying ventricular dilatation and cystic cavity but also the bony defect.

While a growing fracture always implies severe trauma at a time of maximum growth of both the skull and the underlying brain we do not know why the fracture should continue to enlarge. One possibility is that leptomeningeal cysts (fluidfilled spaces between the pia mater and the arachnoid membrane) cause the bone erosion and enlargement. These cysts are, however, rare, and at operation the pia and arachnoid membranes are generally found to be matted together and stuck over the underlying atrophic brain. ${ }^{9}$ More probably the fracture continues to grow both because ventricular size is increasing and because the dura is torn. Nevertheless, the relative contribution of these two factors is not clear and the evidence from experimental work is conflicting. ${ }^{1011}$ After a head injury, changes occur in the dynamics of formation and flow of cerebrospinal fluid, and these may increase its pressure. If the ventricle is enlarged any increase in this pressure will produce a disproportionate rise in the subdural stress; and with a dural defect this increase in stress will cause a pulsatile swelling, which will then erode the bone and increase the defect.

The growth of the fracture, then, results partly from the dural defect. Hence the key to its control is an operation to ensure a watertight closure of the dura. The bony defect itself can then be closed with a split rib graft or with acrylic (which has been used more often in recent years). The outlook after surgery is good: of eight children followed for up to 10 years, five were normal, two had a mild hemiparesis, and only one was slightly mentally backward. The earlier the diagnosis is made the better, but since such fractures are rare repeated radiographs are not justified as a routine in the follow-up of every skull fracture in childhood. Suspicion is indicated only in infants with a parietal fracture with notable separation of the bone edges.

\footnotetext{
${ }^{1}$ Howship, J, Practical Observations in Surgery and Morbid Anatomy, p 494. London, Longman, Hurst, Reece, Ormond-Brown, 1816.

2 Bilroth, T, Archiv für Klinische Chirurgie, 1862, 3, 398.

3 Conner, P S, American fournal of the Medical Sciences, 1884, 88, 103.

4 Trotter, W A, A System of Surgery, ed C C Choyce and J M Beattie, vol 3, p 536. New York, Hoeber, 1923.

5 Dyke, C G, Diagnostic Roentgenology, ed R Golden, p 116. Baltimore, Williams and Hopkins, 1948.

${ }^{6} \mathrm{Pia}, \mathrm{H} \mathrm{W}$, and Tonnis, W, Zentralblatt für Neurochirurgie, 1953, 13, 1.

7 Lende, R A, and Erickson, T C, fournal of Neurosurgery, 1961, 18, 479.

8 Taveras, J M, and Ransohoff, J, Fournal of Neurosurgery, 1953, 10, 233.

${ }^{-}$Kingsley, D, Till, K, and Hoare, R, Fournal of Neurology, Neurosurgery and Psychiatry, 1978, 41, 312.

10 Goldstein, F, et al, fournal of Neurosurgery, 1967, 27, 541.

11 Lende, R A, Neuroradiology, 1974, 7, 119.
}

\section{Quest for excellence}

It looks as though we have got postgraduate medical education in Britain about right. There are, of course, things that need watching. Some training programmes are too inflexible, and general practitioners have set their own precedent by having theirs embodied in an Act of Parliament. The method of assessing posts for training has proved expensive and time 
consuming. There are still inadequate facilities for career guidance. Nevertheless, postgraduate education has been a striking success, and it should have freed the undergraduate curriculum for its rightful job of training the mind. Despite this, however, the indications are that this has not yet happened.

As the result of a close personal look at the system, ${ }^{1} \mathrm{Sir}$ George Pickering is obviously unhappy at the way most undergraduate medical students are taught. A major problem is that there is so much to learn. Thirty years ago there were 14 separate departments headed by a professor; today the average is 30 . Replacing examinations by continuous assessment in some schools (frequently introduced at the request of the students themselves) has become a tyranny: at Birmingham, for example, students have up to 60 assessments during their five-year training. Emphasis on the multiple choice questions in examinations may penalise the brilliant or eccentric student because the questions represent prevailing dogma rather than absolute truth. More damagingly, literacy and scholarship are destroyed: "All we have to do . ..." one student told Sir George, "is to put ticks in the right boxes." For most students "the medical curriculum is virtually as it was thirty years ago and they find it boring and frustrating." Little attempt is made to train them "in curiosity, awareness, precision of thought and expression, and in methods of collecting and assessing evidence." And this at a time when the quality of young persons coming into medicine is higher than it has ever been. Expanding numbers have not helped: the individual student in a large medical school has little opportunity to acquire the mental excitement and enthusiasm for learning which come from close contact with his teachers. The teachers themselves, overwhelmed by numbers, fall back on the formal lecture. This is likely to be particularly disastrous in the introductory clinical course, where mastery of clinical methods is so important. "Today it is mostly badly done."

What are the solutions? Sir George believes that medical students need educating in depth, for example, by doing a BSc or its equivalent, learning by active participation, and above all a change in the attitude of teachers, "to get the student to do the work." This he regards as more important than any changes in the curriculum. MCQ examinations need demystifying and replacing by some method of testing the student's ability to assemble and act on the evidence, in both writing and speaking. Above all, the decline in literacy and scholarship must be halted. Sir George would be the first to admit that his ideas are based on experiments which are already being successfully carried out by a variety of medical schools. It would be unfair to paint the picture too black, but if his excellent report has the same catalytic effect on undergraduate training as his efforts for postgraduate education had in the 'sixties, then the future health of medicine will be assured.

1 Pickering, G, Quest for Excellence in Medical Education. Oxford University Press for the Nuffield Provincial Hospitals Trust, 1978.

\section{The continent urostomy}

Every patient who has had a cystectomy has to face the formidable problems associated with urinary diversion. As the procedure becomes more and more routine, long-term survival is now frequent, particularly in patients with congenital deformities such as ectopia of the bladder. The challenge has stimulated surgeons to devise operations which preserve their patients' renal function and health while causing the minimum possible disturbance to their life. Much of this effort has gone into devising alternatives to an external appliance to collect the urine.

Few would dispute that the most reliable method of preserving renal function, particularly in kidneys already damaged by obstruction or infection, is the ileal conduit pioneered by Bricker. ${ }^{1}$ The conduit empties into an appliance, with its inevitable skin problems and leakages and the need for stoma care, all of which cause distress and difficulty for many patients. Full rehabilitation can be achieved, with good quality long-term results; but a life dependent on an appliance has inescapable problems. It needs understanding and enthusiasm from the patient-and help and acceptance from his family. With good care of the skin and stoma (under the supervision of a stoma-therapist) the appliance will often remain in place for five days to a week at a time. Drainage bags are now satisfactory, though special arrangements may need to be made for drainage at night. There are obvious limitations-contact sports, for example, are not possiblebut the patient can enjoy swimming, skiing, and many other activities.

The alternative of intact ureterosigmoidostomy also offers a good quality of life to patients suitable for the procedure. Normal upper urinary tracts are a prerequisite, but there is always a risk of complications. Even in patients who have survived 40 years or more, reflux and infection can lead to calculus disease and pyelonephrosis, with loss of the kidneys. ${ }^{2}$ With increasing age rectal continence becomes less dependable, and leakage may be troublesome.

Gilchrist and his colleagues ${ }^{3}$ pioneered attempts to construct a continent artificial bladder that would require no appliance. These first attempts in Chicago were synchronous with Bricker's ${ }^{1}$ work in St Louis in developing the appliancedependent conduit. Experience worldwide over thirty years (both types of operation were first reported in the same year) underlines the success of the Bricker operation in many hands. Only recently has interest in the continent urostomy returned. There has been no lack of research effort and the results have been fully recorded. Should surgeons start to use this technique more widely in view of its potential benefits?

If the patient is to have a urinary diversion with no appliance his renal function must be preserved and the upper urinary tract must be protected from reflux. Moreover, the management of the urinary reservoir must be simple enough for the patient himself. The ileocaecal segment of the bowel is ready made for constructing a continent reservoir, the ureters being implanted into the ileal tail; but probably no more than half of all ileocaecal valves are naturally competent. ${ }^{4}$ The alternative is for the ureters to be implanted by an antireflux technique, but ureteric stenosis and upper tract obstruction then become a risk.

The pioneering work of Gilchrist and Merricks and their colleagues was reviewed in 1973 by Sullivan et al. ${ }^{5}$ Forty patients had had an artificial bladder constructed and 37 had achieved complete continence. All but three had had malignant pelvic disease. The patients had to catheterise themselves at four-to-six-hourly intervals in the day and often once at night. In this group an antireflux technique was used in implanting the ureters but reflux was found in three of the 38 patients investigated. No calculi were found, but one patient had serious renal infection. While only six patients survived for a long time (13-20 years), those who died had succumbed to recurrent carcinoma and not renal failure. Successful results have also been reported from 